Pesq. Vet. Bras. 29(4):327-332, abril 2009

\title{
Veias do sistema porta-hepático em gansos domésticos ${ }^{1}$
}

\author{
Tatiana C. Santos ${ }^{2}$, Carla C.F. Borba ${ }^{3}$, Anita Menconi ${ }^{3}$, Marina O. Maia ${ }^{4}$, \\ Pedro P. Bombonatto ${ }^{*}$ e Cheston C.H. Pereira ${ }^{6}$
}

\begin{abstract}
Santos T.C., Ferrari C.C., Menconi A., Maia M.O., Bombonato P.P. \& Pereira C.C.H. 2009. [Veins from hepatic portal vein system in domestic geese.] Veias do sistema porta-hepático em gansos domésticos. Pesquisa Veterinária Brasileira 29(4):327-332. Departamento de Cirurgia, Faculdade de Medicina Veterinária, Universidade de São Paulo, Av. Prof. Dr. Orlando Marques de Paiva 87, Cidade Universitária, São Paulo, SP 05508-270, Brazil. E-mail: bombonat@usp.br

The intraparenchymal distribution of the hepatic portal veins in 30 domestic geese were studied. Stained Neoprene latex was injected into the isquiatic vessels, and the animals were fixed in $10 \%$ formaldehyde by immersion and intramuscular injection. The liver of geese was composed of a large right and a smaller left hepatic lobe, connected by a parenchyma bridge. The right hepatic lobe had vessels exclusively from the hepatic portal system composed by intraparenchymal distribution of the right hepatic portal vein, while the vessels of the left hepatic lobe came from the right hepatic portal vein and from small left hepatic portal veins. The right hepatic portal vein emitted the right caudal branch, which emitted a small right caudolateral branch and a large right caudomedial branch. Cranially this vein emitted right cranial and right lateral branches. The tranverse portion of the right hepatic portal vein crossed to the left hepatic lobe, emitting 1 to 6 small cranial and caudal branches to the medial area of the liver. In the left hepatic lobe, the left branch from the right hepatic vein emitted the left cranial, left lateral and left median branches. One to six left hepatic portal veins were identified arising from the left branch or from the transverse portion of the right hepatic portal vein. These vessels arose from the gizzard and proventricle. In $40 \%$ of geese one proper hepatic portal vein originated from venous vessels of the gizzard and was distributed into the caudal extremity of the left hepatic isolated lobe.
\end{abstract}

INDEX TERMS: Morphology, hepatic portal vein, liver, geese.

RESUMO.- A distribuição intraparenquimal das veias porta-hepáticas foi estudada em 30 gansos domésticos. Latex Neoprene corado foi injetado pela veia isquiática e os animais forma fixados por imersão e injeção intramuscu-

\footnotetext{
${ }^{1}$ Received on April 11, 2008.

Accepted for publication on February 27, 2009.

2 Departamento de Zootecnia, Universidade Estadual de Maringá, Av. Colombo 5790, Maringá, PR 87030-030, Brasil.

${ }^{3}$ Veterinária autônoma, Residente em Ornitopatologia, Faculdade de Medicina Veterinária e Zootecnia (FMVZ) Universidade Estadual Paulista (Unesp), Botucatu, SP.

${ }^{4}$ Graduando em Medicina Veterinária, FMVZ, Universidade de São Paulo (USP), São Paulo.

${ }^{5}$ Departamento de Cirurgia, FMVZ-USP, Cidade Universitária, Av. Prof. Dr. Orlando Marques de Paiva 87, São Paulo, SP 05508-270, Brasil. *Corresponding author: bombonat@usp.br

${ }^{6}$ Centro Regional Universitário de Espírito Santo do Pinhal, Av. Hélio Vergueiro Leite $\mathrm{s} / \mathrm{n}$, Jardim Universitário, Cx. Postal 5, Espírito Santo do Pinhal, SP 13990-000, Brasil.
}

lar com formol a $10 \%$ e dissecados. O fígado esteve composto por um grande lobo hepático direito e por um lobo hepático esquerdo menor, os quais estiveram conectados por uma ponte de parênquima. O lobo direito do fígado teve exclusivamente vasos do sistema porta-hepático formados pela distribuição intraparenquimal da veia portahepática direita, enquanto que no lobo esquerdo estes originaram-se da veia porta-hepática direita e de pequenas veias porta-hepáticas esquerdas. A veia porta-hepática direita emitiu o ramo caudal direito, que emitiu um pequeno ramo caudolateral direito e um grande ramo caudomedial direito. Cranialmente esta veia emitiu os ramos craniais direito e ramos lateral direito. A porção transversa da veia porta-hepática direita cruzou para o lobo hepático esquerdo, emitindo de 1 a 6 pequenos ramos craniais e caudais para a região média do fígado. No lobo esquerdo, o ramo esquerdo da veia porta-hepática direita emitiu o ramo cranial esquerdo, o ramo lateral esquerdo e 
o ramo medial. De 1 a 6 veias porta-hepáticas esquerdas foram identificadas desembocando ou no ramo esquerdo da veia porta-hepática direita ou em sua porção transversa, oriundos do ventrículo gástrico e do pró-ventrículo. Em $40 \%$ dos gansos uma veia porta-hepática própria oriunda da confluência de vasos venosos da face esquerda do ventrículo distribuiu-se na extremidade caudal do lobo esquerdo isoladamente.

TERMOS DE INDEXAÇÃO: Morfologia, veia porta-hepática, fígado, gansos.

\section{INTRODUÇÃO}

O fígado é a maior glândula no organismo e ocupa a porção ventral das regiões cranial e média do corpo das aves. Como em outros vertebrados, o fígado em aves funciona como glândula exócrina via sistema de ductos e como glândula endócrina secretando substâncias diretamente no sangue (McLelland 1989). As funções do fígado podem ser divididas em: funções vasculares, de armazenamento e filtração do sangue; função secretora, produzindo bile para o tubo digestório, necessárias para a emulsificação de gorduras; e função metabólica de carbohidratos, proteínas e gorduras. A composição da bile das aves é semelhante a dos mamíferos, e sua excreçao pode ser realizada diretamente no duodeno ou ser armazenada em uma vesícula biliar (Kolb 1984).

Nas aves o fígado está composto por dois lobos, o direito e o esquerdo, que se unem cranialmente na linha média. O lobo esquerdo, com a forma de prisma, é normalmente menor do que o lobo direito e estende-se, na fêmea, entre os níveis da terceira vértebra torácica e a quarta vértebra lombossacral (McLelland 1986). O lobo direito é mais largo, apresenta vesícula biliar em sua superfície visceral e é atravessado pela veia cava caudal (Dyce et al. 1996). Os ductos biliares e os vasos sanguíneos penetram no fígado em uma fissura transversal da face visceral. A vesícula biliar fusiforme está presente na galinha, no pato e ganso e encontra-se ausente no pombo (Dukes et al. 1996) e na galinha de angola (Kolb 1984). Lucas \& Denington (1956) descreveram divisões centrais dos lobos direito e esquerdo.

O sistema porta-hepático das aves possui algumas diferenças fundamentais daquele dos mamíferos. Este sistema anastomosa-se com o sistema porta-real através da veia coccígeo mesentéricas e apresenta pluralidade na sua formação constituindo dois sistemas, o direito e o esquerdo (Pavoux \& Jolly 1968). Os vasos sangüíneos aferentes do fígado são artérias hepáticas direita e esquerda e as veias porta-hepáticas direita e esquerda (Baumel 1986). A veia porta-hepática direita drena o sangue do duodeno, do pâncreas, do íleo e dos cecos através da veia gastropancreaticoduodenal; do jejuno, íleo e dos cecos através da veia mesentérica cranial; e do reto através da veia mesentérica caudal, estas duas últimas veias se unem e originam a veia mesentérica comum que conflui na veia porta-hepática direita. A veia mesentérica caudal em sua porção distal sofre anastomose com a veia porta-renal caudal, o que garante uma comunicação direta do sistema porta-hepático com o sistema porta-renal (McLelland 1989). A veia porta-hepática esquerda é muito menor e drena o sangue de partes do estômago através da veia pilórica; veia gástrica esquerda (no número de 1 ou 2); veia gástrica ventral; veia pró-ventricular caudal e ventral (Baumel et al. 1979).

O ganso, considerado uma ave doméstica, possui importância econômica fornecendo carne, penas, ovos entre outros produtos. Apesar de seu valor na produção animal, os estudos morfológicos nesta espécie não são extensos. Com a finalidade de contribuir para preencher esta lacuna este estudo visou identificar as veias porta-hepáticas; reconhecer a distribuição dos vasos no parênquima hepático e descrever sua distribuição intraparenquimal, inferindo suas similaridades com outras aves já descritas.

\section{MATERIAL E MÉTODOS}

Para a realização deste trabalho, 30 gansos (Anser domestica), machos e fêmeas adultos, obtidos em criadouros comerciais, foram eutanasiados por aprofundamento do plano anestésico, através de inalação por clorofórmio. Estes animais tiveram o leito arterial preenchido por Neoprene látex corado, através de injeção deste na artéria isquiática. Após a repleção dos vasos, os animais foram fixados em solução aquosa de formol a $10 \%$ e mantidos nesta solução até a dissecação.

A cavidade celomática foi aberta e o fígado identificado, bem como suas relações com órgãos vizinhos. As artérias e veias hepáticas foram identificadas e estabelecidas suas origens junto a outros vasos. O fígado foi então identificado e isolado, para posterior dissecação, pela face visceral, dos vasos sangüíneos e sua distribuição no parênquima hepático e cada vaso foi identificado e estabelecido seu território de responsabilidade. A terminologia adotada está embasada na Nomina Anatomica Avium (Baumel et al. 1979).

\section{RESULTADOS}

O fígado de ganso apresentava a fresco, coloração castanho escura e consistência firme. Macroscopiamente estava composto por dois lobos, um lobo hepático direito maior e por um lobo hepático esquerdo menor, os quais estavam conectados por uma ponte de parênquima. $\mathrm{O}$ lobo direito é proporcionalmente maior (Fig.1), porém em alguns casos ambos os lobos possuíam semelhante proporção (Fig.1B). Posicionado na face ventral da cavidade celomática, em posição anatômica, a inclinação do eixo maior do fígado possuía correspondência com o eixo crânio-caudal do corpo das aves, determinando a nomenclatura hepática para seus lobos e correspondentes vasculares.

Na face côncava do fígado do ganso penetraram os vasos sangüíneos constituintes aferentes do sistema porta-hepático: veia porta-hepática direita, veias porta-hepáticas esquerdas e, em alguns casos, a veia porta-hepática própria. Ainda nessa face observava-se a vesícula biliar e a confluência dos ductos hepáticos, próximo ao hilo hepático. 

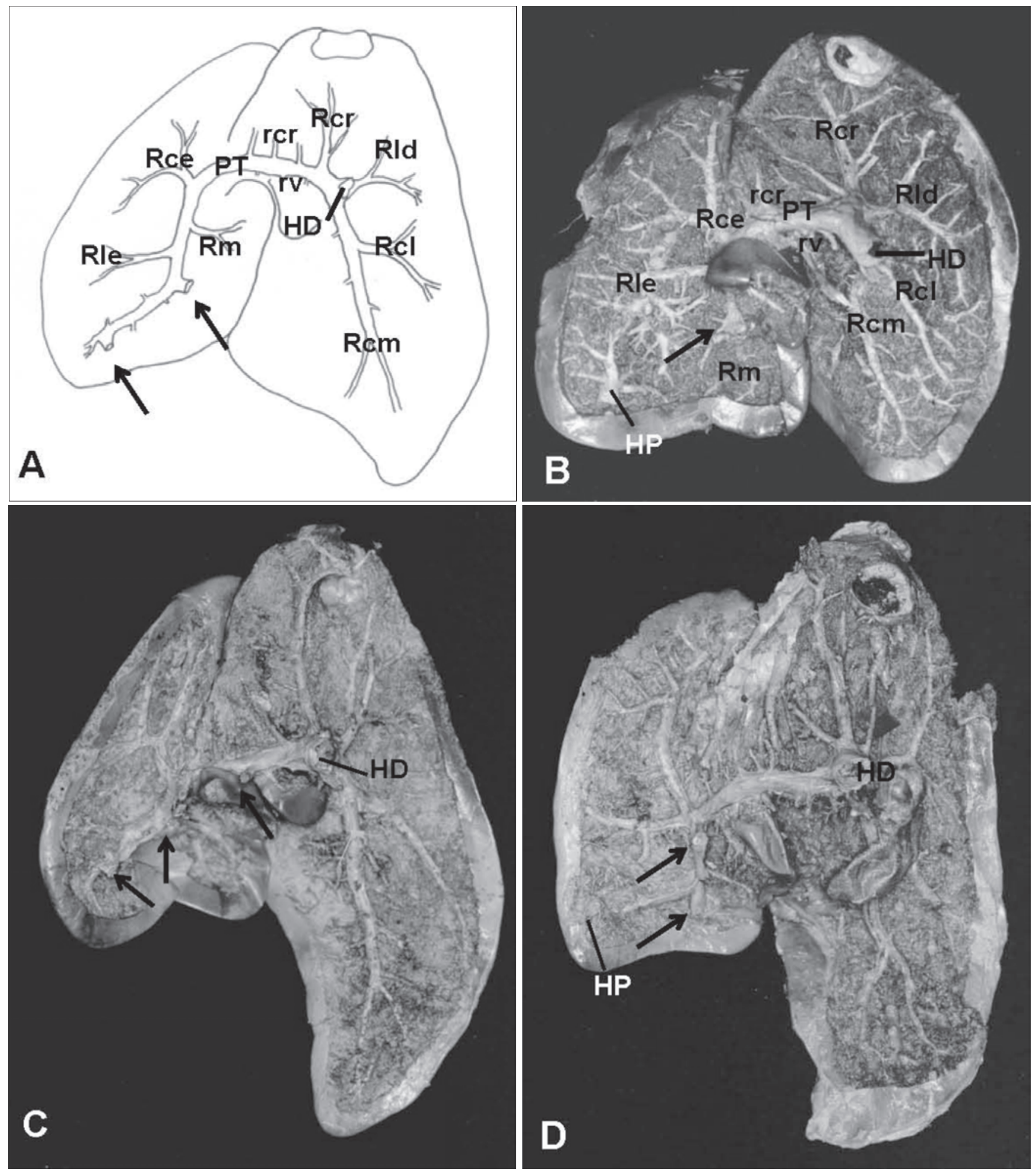

Fig.1. (A) Desenho esquemático da distribuição intraparenquimal das veias porta-hepática no ganso doméstico (distribuição mais freqüente). (B-D) Face visceral do fígado de ganso dissecado com os ramos das veias porta-hepáticas. (HD) Veia portahepática direita, (Rcr) ramo cranial, (Rld) ramo lateral direito, (Rcl) ramo caudolateral, (Rcm) ramo caudomedial, (PT) porção transversa do ramo esquerdo da veia porta-hepática direita, (rv) ramos ventrais da porção transversa, (rcr) ramos craniais da porção transversa, (setas) veias porta-hepáticas esquerdas, $(\mathrm{Rm})$ ramo medial, (Rce) ramo cranial esquerdo, (Rle) ramo lateral esquerdo e (HP) veia porta-hepática própria. 
A distribuição da veia porta-hepática direita no parênquima foi distinta. Mais calibrosa que as demais veias porta-hepáticas, este vaso estava dividido em dois grossos ramos. O ramo caudal direito direcionado caudalmente no lobo hepático direito, emitia um pequeno ramo caudolateral (observado em 25 animais, 83,33\%) e um grande ramo caudomedial (27 animais, 90\%), o qual também se subdividia.

Também da veia porta-hepática direita surgiram os ramos dorsal e lateral direito. O ramo cranial da veia portahepática direita apresentava duas apresentações. Na primeira delas (20 animais, 66,67\%) este vaso era único e a porção do parênquima dorsal que fora responsabilidade do ramo dorsal, passava a ser irrigado por vasos oriundos do ramo lateral da veia porta-hepática direita. Já na segunda forma de apresentação (10 animais, 33,33\%) dois ramos (lateral e medial) surgiram de forma independente, dirigindo-se ao parênquima dorsal do lobo direito do fígado. O ramo lateral direito da veia porta-hepática direita emergia da face lateral da veia dirigindo-se ao parênquima lateral do lobo hepático direito (Fig.1B), ora com alguns ramos ao tecido localizado dorsalmente.

Em direção ao lobo esquerdo do fígado, a veia portahepática emitia um grosso ramo esquerdo, que ao passar pela porção média do fígado formava a porção transversa. Desta porção transversa partiram vários pequenos ramos denominados ramos craniais e caudais da porção transversa, para a região média dorsal do fígado (Fig.1D). Foram observados de 1 a 7 destes vasos em direção cranial $(1,2,3,4,5,6$ e 7 vasos em 5, 3, 6, 7, 4, 1 e 1 animais, respectivamente) e de 1 a 7 vasos em direção caudal $(1,2$, $3,4,6$ e 7 vasos em 4, 6, 4, 6, 2 e 2 animais, respectivamente).

O lobo esquerdo do fígado possuía distribuição intraparenquimal de vasos oriundos da veia porta-hepática direita e por várias pequenas veias porta-hepáticas esquerdas. A porção transversa da veia porta-hepática direita continuava-se como ramo esquerdo no lobo esquerdo do fígado, emitindo o ramo cranial, o ramo lateral e o ramo medial esquerdos. O ramo cranial esquerdo apresentava configurações distintas podendo ser único, bifurcar-se ou ainda trifurcar-se, sendo estas ramificaçoes destinadas a extremidade cranial e lateral do lobo hepático esquerdo. Em 21 gansos suas ramificações mais laterais destinavam-se as porções laterais e caudais do lobo hepático esquerdo. Em 11 animais surgia o ramo lateral esquerdo responsável pelo parênquima lateral do lobo hepático esquerdo, naqueles animais em que o ramo cranial não se ramificava nesse sentido. $O$ ramo esquerdo da veia porta-hepática direita emitia para a porção medial do lobo hepático esquerdo dois ou três ramos mediais em todos os animais analisados.

As veias porta-hepáticas esquerdas surgiam da confluência de pequenas veias oriundas da curvatura menor do ventrículo e do pró-ventrículo gástricos, e distribuíam pelo parênquima do lobo hepático esquerdo na face côncava deste, após desembocarem no ramo esquerdo da veia porta-hepática direita ou em sua porção transversa. Esses vasos variaram em número de 1 a 6 , sendo que em aves com maior número de vasos o calibre destes estava reduzido proporcionalmente aquelas aves em que estes confluíam para um vaso único antes de penetrar no parênquima. A freqüência do número de veias porta-hepáticas esquerdas observados era de 1, 2, 3, 4, 5 e 6 vasos respectivamente em 5, 3, 8, 8, 3 e 2 aves, sendo, portanto, 3 e 4 veias o número mais freqüentemente observado.

Ainda sobre o lobo esquerdo do fígado de gansos, particularmente em sua extremidade caudal, em 12 gansos (40\%) observava-se uma veia porta-hepática própria, oriunda da confluência de vasos venosos da face esquerda do ventrículo gástrico, sem conexões macroscópicas com o restante do sistema porta-hepático, constituindo um sistema porta-hepático independente (Fig.1B, 1D).

Apesar dos ramos formadores das veias hepáticas não serem objetos deste estudo, estes foram observados confluindo em direção dorso-cranial para a margem dorsal do lobo direito do fígado, formando a veia hepática que desembocava na veia cava caudal.

\section{DISCUSSÃO}

Os aspectos macroscópicos do fígado de gansos reforçam as descrições que sugerem um padrão de apresentação do fígado nas aves, em que o órgão compõe-se por dois grandes lobos unidos medianamente por uma ponte de parênquima. Para McLelland (1989) a proporção entre os 2 lobos hepáticos é descrito como homogênea em emas, enquanto que na maioria das aves o lobo direito é proporcionalmente maior que o esquerdo. Também em gansos observou-se o lobo direito proporcionalmente maior, apesar de em algumas aves essa proporção ter sido reduzida.

Nos gansos o sistema porta-hepático está composto pela veia porta-hepática direita, por várias (1 a 6) pequenas veias porta-hepáticas esquerdas e em alguns gansos por uma veia porta-hepática própria, presente na extremidade caudal do lobo hepático esquerdo. A maioria dos autores citam duas veias porta-hepáticas, sendo a direita a principal drenando o trato digestório e a esquerda drenando parte do ventrículo gástrico (Baumel 1986, McLelland 1989, Dyce et al. 1996, Pinto et al. 1999). Em alguns trabalhos mais detalhados (Pavaux \& Jolly 1968) a veia porta-hepática própria também foi citada.

Em relação à distribuição intra-hepática da veia portahepática direita, os padrões de distribuição e suas variações são citados em aves domésticas por Pavaux \& Jolly (1968) e Miyaki (1972). Sobre a nomenclatura intraparenquimal desses ramos oriundos do sistema porta-hepático, alguns autores utilizaram os termos cranial e caudal, ou anterior e posterior (Pavoux e Jolly 1968). Nesse trabalho os termos seguiram os apresentados na Nomina Anatomica Avium (Baumel et al. 1979), em que são citados os termos cranial e caudal, em virtude da posição anatômica das aves e da posição do fígado na cavidade celomática, termos estes também adotados nas descrições de Miyaki (1972). 
A porção transversa da veia porta-hepática direita pode ser encontrada com diferentes denominações na literatura, entre eles ramo comunicante (Miyaki 1972) ou anastomose portal intersinusal (Pavoux \& Jolly 1968). Particularmente sobre o último termo discorda-se dessa terminologia por este vaso não se tratar de uma anastomose, mas sim de uma ramificação em sentido esquerdo, a partir da veia porta-hepática direita, em direção ao lobo hepático esquerdo. Outro fato que corrobora esse ponto é que esse vaso diminui seu calibre em sentido ao lobo esquerdo e mesmo nos pontos de confluência das veias porta-hepáticas esquerdas, o vaso permanece com calibre mais espesso que estas, e desta forma o sangue proveniente destas pequenas veias mistura-se com aquele proveniente da veia porta-hepática direita antes de distribuir-se pelo parênquima hepático.

Com relação a veia porta-hepática própria, Pavaux \& Jolly (1968) descrevem este vaso como padrão para os gansos, denominando-a de veia porta-hepática "independente". No entanto, nossos resultados demonstram que em alguns casos (60\%) o vaso venoso oriundo da face esquerda do ventrículo gástrico e/ou do pró-ventrículo unese aos demais comunicando-se com o sistema vascular que supre o lobo esquerdo contribuindo para a formação do ramo esquerdo. Em 12 animais (40\% dos animais) este vaso apresenta-se isolado constituindo um sistema porta, porquanto, antes de alcançar o parênquima hepático, os vasos que o formam constituem um tronco para, a seguir, ao penetrar no parênquima, dividir-se na região caudal do lobo esquerdo.

A proximidade do fígado com o ventrículo gástrico é reforçada pela presença das veias que partem na face ventral deste órgão em direção ao fígado, as veias porta-hepáticas esquerdas. Nos gansos estudados um variado número desses vasos foram encontrados, sendo os mais freqüentes com 3 e 4 veias porta-hepáticas esquerdas oriundas das faces cranial e ventral do ventrículo gástrico. Sobre este aspecto, nas galinhas cita-se uma veia porta-hepática esquerda drenando o sangue do território suprido pelo ramo esquerdo da artéria celíaca. Nesta espécie este vaso forma-se ventralmente a junção pró-ventricular pela união das veias gástricas ventral, gástrica esquerda e próventricular caudal (Nishida et al. 1969). Pavoux \& Jolly (1968) citam veias porta-hepáticas secundárias drenando o estômago de aves entrando no fígado em sua face visceral esquerda, em pombos um único vaso e em galinhas e anseriformes de 3 a 5 vasos podem ser encontrados. Já nos patos, Pinto et al. (1999) destacam que apenas uma veia porta-hepática esquerda penetra no fígado formada por vasos provenientes do ventrículo, pró-ventrículo e piloro. Apesar de em alguns animais terem sido encontrada apenas uma veia porta-hepática esquerda, esta não foi a forma mais freqüente nos gansos estudados. Em pombos as veias proventricular caudal e gástricas esquerda e gástrica ventral confluem para uma veia porta-hepática esquerda, enquanto as veias gástricas direitas desembocam na veia porta-hepática direita (Malinovsky 1965).
Sobre estes vasos formadores da veia porta-hepática esquerda Diaconescu (1971) possui uma teoria interessante. Segundo ele em vertebrados inferiores o fígado recebe sangue aferente visceral e parietal da parede do tronco. Nos peixes o fígado recebe sangue das veias hepatointestinais e subintestinais. O sangue parietal que chega ao fígado provém de veias cardinais posteriores que se anastomosam com a veia intestinal e não possuem sistema porta-renal. Já em anfíbios o fígado recebe sangue visceral gastroenterolienal pela veia porta visceral e sangue parietal das veias abdominais e veias ilíacas. Em répteis as veias parietais estão reduzidas em comparação aos anfíbios e em aves os vasos são predominantemente viscerais e o sangue parietal é drenado para a veia cava. Os vasos que drenam a parede muscular do ventrículo gástrico das aves seriam os representantes dessa circulação e corresponderiam as veias porta-hepáticas esquerdas descritas nos gansos no presente trabalho.

Ainda segundo Diaconescu (1971), o fenômeno de o fígado receber sangue parietal diminui progressivamente quando se analisam as classes de animais, podendo ser encontrados apenas rudimentos nos mamíferos e homem. Esse processo evolutivo é conhecido como visceralização do fígado e ocorre concomitantemente com a aquisição da capacidade de neoglicolização dos músculos somáticos nos vertebrados.

O sistema porta-hepático carreia os nutrientes e outras substâncias absorvidas pelo intestino, pâncreas, estômago e regiões do intestino grosso (ceco e reto) (Nickel et al. 1977), este fator colabora para que substâncias encontradas na ração sejam facilmente metabolizadas, porém, quando temos fatores tóxicos nos alimentos ou injetados nos membros, a toxicidade é mais rápida devido à comunicação com o sistema porta-renal (Benez 1986).

O preenchimento das veias porta-hepáticas, após injeção de Neoprene látex via veia isquiática, deve-se provavelmente as anastomoses entre vasos que formam a veia porta-hepática direita e as veias renais, e anastomoses entre o sistema porta renal e as veias ilíacas, deixando os dois sistemas portais, hepático e renal, conectados. Este fenômeno é citado de uma forma geral em aves (Nickel et al. 1977, Baumel 1986, West et al. 1989). Em galinhas (Nishida et al. 1969) cita-se ainda uma rede venosa com comunicação direta entre a circulação sistêmica e a portal a partir do ventrículo gástrico, que possui drenagem venosa em sentido da veia porta-hepática direita, esquerda e diretamente na veia cava caudal.

Por fim as observações feitas por Miyaki (1978) em das galinhas, citando que em $48 \%$ das aves possuiam pequenas veias aferentes entrando nas margens do fígado, vindas dos sacos aéreos e do peritônio. Sobre este aspecto Nickel et al. (1977) comentam que a veia porta-hepática coleta sangue do canal gastrointestinal, pâncreas, baço e parte dos sacos aéreos. Estas observações não foram realizadas, porém não se descarta a possibilidade de ocorrência em gansos.

Após estas considerações pode-se concluir que o fí- 
gado dos gansos domésticos mantém um padrão de distribuição intra-hepática de seus vasos semelhante a de galinhas e patos, com variações principalmente no que se refere a formação de várias veias porta-hepáticas esquerdas, a partir de sangue drenado do ventrículo gástrico, e a presença de uma veia porta-hepática própria em alguns animais, formando um sistema macroscopicamente independente.

\section{REFERÊNCIAS}

Baumel J.J. 1986. Coração e vasos sangüíneos das aves, p.1842-1880. In: Getty R. (Ed.), Sisson and Grossman, Anatomia dos Animais Domésticos. Vol.2. Guanabara Koogan, Rio de Janeiro.

Baumel J.J., King A.S., Lucas A.M., Breazile J.E. \& Evans H.E. 1979. Nomina Anatomica Avium: An annoted anatomical dictionary of birds. Academic Press, London.

Benez S.M. 1986. Aves: Criação, clínica, teoria, prática. Robe Editorial, São Paulo, p.37-39.

Diaconescu N. 1971. On the liver "visceralisation" in the vertebrate series. Acta Anat. 78:74-83.

Dukes H.H., Swenson M.J. \& Reece W.O. 1996. Fisiologia dos Animais Domésticos. Guanabara Koogan, Rio de Janeiro

Dyce K.M., Sack W.O. \& Wensing, C.J.G. 1996. Anatomia das aves, p.631-650. In:___(Eds), Tratado de Anatomia Veterinária. Guanabara Koogan, Rio de Janeiro.

Kolb E. 1984. Fisiologia Veterinária. Guanabara Koogan, Rio de Janeiro. p.191-197.
Lucas A.M. \& Denington E.M. 1956. Morphology of the chicken liver. Poultry Sci. 35:793-806.

Malinovsky L. 1965. A contribution to the comparative anatomy of vessels in the abdominal part of body cavity in birds. II. A comparison of the vascular to the stomachs and adjacent organs of the buzzard (Buteo buteo L.) and domestic pigeon (Columbia livia L., f. domestica). Folia Morphol. 13:202-211.

McLelland J. 1986. Sistema digestivo das aves, p.1740-1763. In: Getty R. (Ed.), Sisson and Grossman, Anatomia dos Animais Domésticos. Vol.2. Guanabara Koogan, Rio de Janeiro.

McLelland J. 1989. Digestive system, p.69-181. In: King A.S. \& McLelland J. (Eds), Form and Function in Birds. Vol.2. Academic Press, London.

Miyaki T. 1972. The hepatic lobule and its relation to the distribution of blood vessels and bile ducts in the fowl. Jap. J. Vet. Sci. 35:408-415.

Nickel R., Schummer A. \& Seiferle E. 1977. Anatomy of the Domestic Birds. Vol.1. 4th ed. Verlag Paul Parey, Berlin, p.518.

Nishida T., Paik Y. \& Yasuda M. 1969. Comparative and topographical anatomy of the fowl. LVIII. Blood vascular supply of the glandular stomach (ventriculus glandularis) and the muscular stomach (ventriculus muscularis). Jap. J. Vet. Sci. 31:52-70.

Pavaux C.L. \& Jolly A. 1968. Note sur la structure vasculo-canaliculaire du foie des oiseaux domestiques. Revue Méd. Vét. 119(5):445-466.

Pinto M.R.A., Ribeiro A.A.C.M., Souza W.M., Miglino M.A. \& Machado M.R.F. 1999. Estudo do sistema portal hepático no pato doméstico (Cairina moshata). Braz. J. Vet. Res. Anim. Sci. 36(4):173-177.

West N.H., Langille B.L., Jones D.R. 1989. Cardiovascular system, p.235339. In: King A.S. \& McLelland J. (Eds), Form and Function in Birds. Vol.4. Academic Press, London. 